\title{
RELATIVISTIC JETS AND COSMIC RAY ACCELERATION
}

\author{
A. Meli $^{1}$
}

\begin{abstract}
Cosmic rays are accelerated in astrophysical plasmas which collide at supersonic speeds where shock waves are formed, and along with other instabilities, they compete for the dissipation and acceleration mechanisms. The diffusive acceleration mechanism plays a leading role in the explanation of very high energy cosmic rays observed. In this mechanism, particles are repeatedly gaining energy in multiple crossings of an astrophysical shock discontinuity, due to collisions with upstream and downstream magnetic scattering centers, resulting in a power-law spectrum extending up to very high energies. Relativistic jets and their shocks in Active Galactic Nuclei (AGN) is a prominent source for particle acceleration. Especially, relativistic single or multiple shocks have been theorized and observed along the jets of AGN and are claimed to be responsible for accelerating even the highest-energy cosmic rays observed. In this paper we will report and discuss the cosmic ray acceleration efficiency and properties of single or multiple shocks in the limit of relativistic plasmas in AGN jet environments.
\end{abstract}

\section{Jets, shocks and particle acceleration}

A favorable source for the acceleration of high energy cosmic rays is believed to be the relativistic shocks in the jets and radio lobes of Active Galactic Nuclei (AGN) (e.g. Ginzburg \& Syrovatskii 1963; Rachen \& Biermann 1993).

It is until now not fully understood to what extent the astrophysical bulk flows are due to leptonic flow (electrons and positrons) or baryonic flow (electrons and ions). It is also not fully understood by which mechanisms this bulk flow energy can be converted into cosmic ray radiation reaching energies of $\mathrm{TeV}$ and beyond. Evidence, in form of power law spectra of the observed cosmic ray radiation over wide energy intervals, favors the Fermi acceleration mechanism at shocks (e.g. Bell 1978), namely the diffusive shock acceleration mechanism, which raises a significant interest in the research community. In the diffusive shock acceleration mechanism particles experience a reflection by the local magnetic field

${ }^{1}$ IFPA, Department of Astrophysics and Geophysics, University of Liege, Belgium 
and large-amplitude magneto-hydrodynamic waves on both sides of a shock. Some particles can bounce back and forth across the shock, and by the velocity difference jump upstream and downstream, they increase their energy at each shock crossing, reaching very high energies with characteristic power-law spectra. This mechanism can take place in more than one consecutive shocks as well.

The jets of AGN black holes are propelled by magnetic fields which are twisted by differential rotation of their central black hole accretion disk which fuels them, or by the inertial frame dragging ergosphere (e.g. Blandford \& Znajek 1977). Observations indicate that AGN jets undergo a very large expansion at the exit from their inner black hole core. In a few parsecs its radius multiplies by more than a factor of a thousand.

State of the art laboratory experiments of supersonic highly conductive plasma flows (e.g. Lebedev et al. 2005; Ampleford et al. 2008) have shown development of oblique or conical shocks forming at the base of a supersonic jet and along its axis. The latest observations have witnessed a development of standing or traveling shocks in many astrophysical objects, and most prominently in AGN jets (e.g. Marscher et al. 2008).

\section{Simulation studies of particle acceleration in relativistic shocks}

There is compelling evidence by observations and theory for ultra-high bulk Lorentz factors in AGN jets (e.g. Begelman et al. 2008; Keppens et al. 2008; Kellerman et al. 2007). Here we will briefly overview a series of numerical studies on relativistic (single or multiple) shock acceleration and their properties, as an application to AGN jets environments.

Before we go into more detail, we note that a multitude of past studies has shown that in non-relativistic shocks the spectral index is independent of the shock inclination to the magnetic field and the scattering of the particles in the fluidrest frames, nevertheless it seems that in relativistic shocks the particle's spectral index becomes a function of the flow speed, the field obliquity, and the nature of the scattering, all of which closely control the degree of particle anisotropy (e.g. Ellison \& Double 2004; Stecker et al. 2007; Meli 2011). It is generally accepted that in parallel relativistic shocks particles boost their energy by a $\Gamma^{2}$ at least (see Achterberg et al. 2001). Moreover, the accelerated particles increase further their energy by a few more $\Gamma$ when the inclination of the shock becomes high enough but not superluminal (further details see in, Meli \& Quenby 2003). Should be noted that there used to be a general belief that a universal powerlaw index of -2.2 must be obeyed by both non-relativistic and relativistic shocks. Nevertheless, it is important to clarify here that such a claim applies only for parallel relativistic shocks and for extremely small pitch angle (or fine) scattering (see discussion in Meli 2011).

In Meli \& Quenby (2003), Meli et al. (2008), Meli (2011), systematic Monte Carlo simulation studies were conducted for individual oblique relativistic shocks,

simulating the AGN jet environments in the test-particle approximation. Among other it was found that oblique relativistic shocks with inclination angles less 
than $45^{\circ}$ to the magnetic field vector of the magnetized plasma, were very efficient in accelerating particles up to $10^{12} \mathrm{GeV}$, factors of $10^{9 \rightarrow 11}$ above the particle injection energy, resulting in flat spectra $E^{-\sigma}$, with spectral index $\sigma$ values ranging between $1.1 \pm 0.1$ and $2.1 \pm 0.1$, while near-perpendicular relativistic shocks were effective only up to $\sim 10^{5} \mathrm{GeV}$, resulting in steeper indices of $\sigma \sim 2.5 \pm 0.1$. Interestingly, it was shown prominently in all simulations, that as relativistic a shock speed goes, as flatter the produced particle power-law spectra, followed by small deviations in the form and inclination of the spectrum, additionally dependent on the level of strength of the particle scattering with the magnetized plasma.

The spectral properties found by the above studies are consistent with observations of gamma-ray spectra supposedly originating by accelerated particles (electrons or protons) from the terminal hotspots and lobes of the powerful FR-II radio galaxies which are not of a single and universal power-law form, as shown for example in detail in Rudnick et al. (1994), Machalski et al. (2007), and others. Regarding direct evidence of acceleration, although there are few FR-II galaxies within the GZK horizon, no correlation has been found between the positions of these objects and the arrival directions of the Auger experiment detections.

Furthermore, in the work of Meli \& Biermann (2012) a numerical simulation model was presented, assuming multiple oblique relativistic shocks in an AGN jet. The model was based on observational indications, assuming acceleration of the same number of test-particles injected once towards a sequence of relativistic shocks with different inclinations to the jet axis, denoting various stages of acceleration or decceleration of the inflow plasma jet. Specifically, six exemplary sets of four inclined shocks were used as case studies calculating particles differential spectra after consecutive acceleration. Particle spectra were recorded for each one of the consecutive multiple shocks for comparison purposes. All spectra manifested a gradual flatness from one shock to another, but with a distinctive gradual decreased flux at the lower energies, with an extension of the particle distributions to higher energies. The behavior of these spectra showed that in a very probable situation of multiple shocks in an AGN, with a fewer number of particles very high energies could be obtained, and this would in turn imply that less source-power would be needed to feed the kinetic energy of the particles. In more detail, it was shown that the first shock of all four-shock sequences develops a quite steep spectrum of $\sigma \sim-2.8$ to -2.7 and the consecutive shocks in the sequence, smooth out and flatten the distribution of the accelerated particles, pushing them into higher energies, some orders of magnitudes above the injection energy.

Specifically, four consecutive near-perpendicular relativistic shocks generated a final particle spectrum registering a spectral index $\sigma \sim-2.3$, and maximal particle energies reaching $10^{8} \mathrm{GeV}$. On the contrary, the set of oblique shocks with inclination less than $45^{\circ}$ to the jet axis, generated very flat spectra with a final value $\sigma \sim-1.4$, and an impressive attained particle energy of $10^{11} \mathrm{GeV}$. Given the ambient photon fields are not very dense in many jets, and the magnetic field strengths weak as many observations indicate, the accelerated protons may escape these regions almost loss-free, subject only to scattering in the permeated intergalactic magnetic fields. 


\section{Conclusion}

Relativistic jets in AGN and their shocks play an important role for the generation of high energy cosmic rays with flat or irregular spectra. An overview over a series of numerical studies was presented here, regarding relativistic particle acceleration properties in single or multiple oblique shocks, as they could occur in relativistic AGN jets. We have confirmed an absent "universal" particle spectrum, compared to the theorized "fixed" spectrum $(\sigma \sim-2.2)$ generated by non-relativistic shocks. A variety of spectral indices depending on shock speeds, with an overall noticeable efficiency and flatness developing at higher shock boost factors was developed through our simulations over almost all cases, for individual or multiple oblique and near-perpendicular shocks. It was made clear that oblique relativistic shocks are very efficient accelerators resulting into very high energy but flat particle spectra. On the other hand highly inclined shocks (superluminal) did not show to be efficient accelerators for the very high energy cosmic rays. It was also shown that in the relativistic speed limits, different shock inclinations give rise to a multitude of spectral slopes as well as some structure in the developed particle distributions, bound on the strength of particle scattering with the magnetized media. It is important to note that our Monte Carlo studies are well compared with similar works of e.g. Ellison \& Double (2004), Niemiec \& Ostrowski (2004), Stecker et al. (2007), etc. These series of simulation studies are important since they shed light into understanding the cosmic ray acceleration mechanism and its properties in the limit of relativistic plasmas in AGN jets, with consequences on our understanding of radiative processes and emission of gamma-rays or neutrinos by extragalactic jets.

\section{References}

Achterberg, A., Gallant, Y.A., Kirk, J.G., \& Guthmann, A., 2001, MNRAS, 328, 393

Ampleford, D.J., Jennings, C.A., Lebedev, S.V., Hall, G.N., et al., 2008, APS, APR8HE013

Begelman, Mitchell, C., Fabian, Andrew, C., \& Rees, Martin, J., 2008, MNRAS, L384, 19B

Lebedev, S.V., Ciardi, A., Ampleford, D.J., Bland, S.N., et al., 2005, PPCF, 47B, 465

Bell, A.R., 1978, MNRAS, 182, 147

Blandford, R.D., \& Znajek, R.L., 1977, MNRAS, 179, 433

Kellermann, K.I., Kovalev, Y.Y., Lister, M.L., et al., 2007, Ap\&SS, 311, 231

Keppens, R., Meliani, Z., van der Holst, B., \& Casse, F., 2008, A\&A, 486, 663

Marscher, A.P. Jorstad, S.G., D'Arcangelo, F.D., Smith, P.S., et al., 2008, Nature, 452, 966

Rachen, J.P., \& Biermann, P.L., 1993, A\&A, 272, 161

Meli, A., \& Quenby, J.J., 2003b, APh, 19, 649

Meli, A., Becker, J.K., \& Quenby, J.J., 2008, A\&A, 492, 323

Meli, A., 2011, Adv. Space Res., 7, 287 
Meli, A., \& Biermann, P.L., 2012, A\&A, in press

Ginzburg, V.L., \& Syrovatskii, S.I., 1963, SvA, 7, 357

Ellison, \& Double, 2004, Astropart. Phys., 22, 323

Rudnick, L., Katz-Stone, D.M., \& Anderson, M.C., 1994, ApJS, 90, 955

Machalski, J., et al., 2007, A\&A, 462, 43

Niemiec, J., \& Ostrowski, M., 2004, ApJ, 610, 851

Stecker, F.W., Baring, M.G., \& Summerlin, E.J., 2007, ApJ, 667, L29 